\title{
Application of $\mathrm{Nano}^{-\mathrm{TiO}_{2}}$ in Cosmetics
}

\author{
Yiwen Fang, ${ }^{*}$ Xupeng Lu, and Wenbin Chen \\ Department of Chemistry, Shantou University, Shantou, Guangdong 515063, China
}

\begin{abstract}
As an inorganic sunscreen material, nano-titanium dioxide $\left(\right.$ nano- $\left.\mathrm{TiO}_{2}\right)$ owns broad prospects in sunscreen cosmetics for its small size, non-toxicity, strong covering ability, UV absorption and scattering abilities, etc. $\mathrm{Nano}^{-\mathrm{TiO}_{2}}$ has high photocatalytic activity, so it will go through a series of complex chemical reactions under light irradiation and cause damage to its surrounding agents. In addition, it is hard to disperse in various aqueous and non-aqueous mediums due to its high surface energy and hydrophobic property. In order to apply nano- $\mathrm{TiO}_{2}$ to cosmetics effectively and safely, its surface modification is necessary. This paper reviews nano- $\mathrm{TiO}_{2}$ 's preparation, modification, and its application in sunscreen cosmetics.
\end{abstract}

Keywords nano- $\mathrm{TiO}_{2}$, preparation, surface modification, application

\section{Introduction}

According to different wavelengths, ultraviolet light (UV) can be divided into ultraviolet radiation C (UVC, wavelength range 200$280 \mathrm{~nm}$ ), ultraviolet radiation B (UVB, wavelength range $280-320$ $\mathrm{nm}$ ) and ultraviolet radiation A (UVA, wavelength range 320-400 $\mathrm{nm}) .{ }^{[1-4]}$ Most UVC in sunlight is absorbed by the ozone layer and can't reach the ground, so it does not harm the human body. However, UVA and UVB may cause serious harm to human skin. UV energy accounts for about $6.1 \%$ of the total solar energy, and UVA energy accounts for $98 \%$ of the total UV energy. The penetration ability of UVA is far stronger than UVB. Most UVA can go through dermal layers, and a small amount of UVA can even go through subcutaneous tissues. Long-term exposure to UVA will gradually destroy blood vessel walls and elastic fibers in connective tissues, which causes further loss of muscle elasticity. Then, skin relaxation, wrinkles, freckles and age spots appear. Most UVB can be absorbed by epidermis, and a small amount of UVB can only go through dermis. Exposure to UVB will cause acute skin erythema or acute dermatitis, known as sunburn. Excessive exposure to UV can also cause skin cancer. There are nearly 500,000 new skin cancer patients in the United States each year. Some researches showed that more than $98 \%$ new skin cancer patients are affected by the sun. ${ }^{[5,6]}$

It is urgent for chemists to take effective measures to shield UV radiation in the sunlight, reduce the damage to the skin greatly caused by UV light, and help the skin to be restored in a short time. In recent years, some researchers in developed countries carried out works on sunscreen products actively. Sunscreen is added to more than $50 \%$ of cosmetics in the United States. ${ }^{[6]}$ Early, organic compounds were utilized in sunscreen cosmetics as UV absorbers, but most of them can only shield UVB, have skin toxicity, and even cause skin allergy. ${ }^{[7,8]}$ In order to avoid the situations, nano- $\mathrm{TiO}_{2}$ is performed as an inorganic UV absorber in sunscreen.

Nano- $\mathrm{TiO}_{2}$ is a small white particle with several advantages such as non-toxic, odorless, hard to decompose under UV irradiation, and hard to react with other chemical agents. It can also shield UVA and UVB effectively. Therefore, nano- $\mathrm{TiO}_{2}$ becomes the world's favorite sunscreen in cosmetics with increase of quantity demand and quality requirements for sunscreen cosmetics. Because of its safety and ef- fectiveness, nano- $\mathrm{TiO}_{2}$ is widely used in sunscreen, lipstick, foundation cream, sunscreen mousse, blemish balm, and other cosmetics. Due to consumers' pursuit of white and tender skin, UV-proof becomes one of basic functions of skin care products. It has maintained a growing trend of using nano- $\mathrm{TiO}_{2}$ in sunscreen cosmetics in the world. ${ }^{[9,10]}$

\section{Preparation of nano- $\mathrm{TiO}_{2}$}

Nano- $\mathrm{TiO}_{2}$ can be prepared by gas, liquid, and solid phase methods. At present, gas and liquid phase methods are mainly employed for preparation of nano- $\mathrm{TiO}_{2}$. Nano- $\mathrm{TiO}_{2}$ prepared by the gas phase method possesses several advantages such as small particle size and good dispersion, but it suffers from large energy consumption and high cost. The liquid phase method is simple, easy to operate and inexpensive, but the morphology of $\mathrm{TiO}_{2}$ is not easy to control.

\section{Gas phase method}

\section{Physical vapor deposition method}

Firstly, raw materials will be converted into gas or plasmas by evaporation, ionization, or sputtering. Then, the gas or plasma will be quenched to form nanoparticles. Nano- $\mathrm{TiO}_{2}$ has characteristics of high purity, uniform distribution, small particle size, and good dispersity. Its particle size and distribution can be controlled by changing gas pressure and heating temperature. ${ }^{[11,12]}$ It was reported that single-crystal $\mathrm{TiO}_{2}$ nanowires were synthesized by a physical vapor deposition (PVD) method. The thickness of nano-film by PVD is uniform and easy-to-control; moreover, its light transmittance and glossiness are also satisfactory. However, the PVD method requires deposition in a vacuum environment and the devices are more expensive. $^{[6,13]}$

\section{Chemical vapor deposition method}

Nano powder is prepared using chemical vapor deposition (CVD) methods using the vapor of volatile compounds as reaction reactants, then reacts and condenses rapidly in the protective gas to get required materials. The fine spherical nano- $\mathrm{TiO}_{2}$ particles prepared by CVD have characters of small size, high chemical activity, well dispersion, good visible light transmittance, strong UV absorb and shielding ability. The CVD process is easy to enlarge and able to use in con-

* E-mail: ywfang@stu.edu.cn; Tel.: 0086-13502753026 Received January 14, 2017; accepted February 24, 2017. 
tinuous production. However, this method requires big one-time investment, while the collection and storage of powder are still difficult. ${ }^{[6,13,14]}$ It was reported that $\mathrm{TiO}_{2}$ crystal films with thickness less than $30 \mathrm{~nm}$ and $\mathrm{TiO}_{2}$ nanocrystals with particle sizes less than $10 \mathrm{~nm}$ were prepared using chemical vapor synthesis (CVS), an improved CVD method. ${ }^{[15,16]}$

\section{Liquid phase method}

Liquid phase methods have been widely utilized to prepare nanoparticles for their simple operations. The nucleation process is very easy to control, additives can be evenly distributed, and high purity composite of nano-oxide can be obtained.

\section{Micro-emulsion and inverse micro-emulsion method}

Micro-emulsion methods for preparing nano- $\mathrm{TiO}_{2}$ are very popular in recent years. The researches on composition, properties, structures of micro-emulsion and how it affects particle sizes and morphology of nano- $\mathrm{TiO}_{2}$ particles are hotspots in the field. ${ }^{[17,18]}$ Micelles are surfactant molecular aggregates dispersed in the aqueous sol, and their concentrations are higher than the critical micelle concentration (CMC). In the micelles, hydrophilic groups in the head converge toward the outside, forming a relatively ordered structure. There are three levels of CMC as follows: CMC-I means the concentration of lipid molecules self-assembled into single-layer spherical micelles, CMC-II refers to the concentration of spherical micelles elongate to form tubular structures, and CMC-III (also known as the lamellar point) refers to the concentration of the stacked tube-forming lamellar structure. CMC depends mainly on chemical compositions of lipid molecules, especially the ratio of head length to tail length.

Reverse micelle refers to an aggregate of surfactant molecules dispersed in a non-aqueous solvent with its hydrophilic head group inward. Unlike micelles, formation of reverse micelle is insensitive to concentration, because a small amount of agglomerates are only formed. Hence, there is no obvious CMC in formation of reversed micelles. It may appear to be a spherical shape even a columnar or oval shape, and it can also form bimolecular layer structures. The shape of reversed micelles depends on geometry of surfactant molecules, concentration, temperature, $\mathrm{pH}$, and ionic strength of solution ${ }^{[6,19]}$ The method requires a calcination process, so the amorphous particles can grow into a crystal with a certain configuration. However, calcinations will cause agglomeration. In order to overcome the drawback, researchers calcined the sample at relatively low temperatures and annealed to maintain micelles' particle size and morphology. ${ }^{[20]}$

Kim et al. ${ }^{[21]}$ did a series of works on optimum synthesis conditions of nano- $\mathrm{TiO}_{2}$. They found that the ratio of $\mathrm{H}_{2} \mathrm{O}$ /surfactant and $\mathrm{H}_{2} \mathrm{O} /$ Ti precursor, ammonia concentration, reactant addition rate and reaction temperature were important factors for controlling $\mathrm{TiO}_{2}$ 's size and size distribution. The crystal structures of products are different at different calcination temperatures. When calcined at $600{ }^{\circ} \mathrm{C}$, amorphous $\mathrm{TiO}_{2}$ nanoparticles with size of $10-20 \mathrm{~nm}$ can be transformed into anatase $\mathrm{TiO}_{2}$. When calcined at $900{ }^{\circ} \mathrm{C}$, more stable rutile nano- $\mathrm{TiO}_{2}$ particles can be obtained thermodynamically. $\mathrm{Li}$ et al. ${ }^{[22]}$ prepared amorphous $\mathrm{TiO}_{2}$ nanoparticles using $\mathrm{TiCl}_{4}$ solution to react with ammonia in a reverse micellar system. The products were transformed into anatase $\mathrm{TiO}_{2}$ during 200 to $750{ }^{\circ} \mathrm{C}$, which were transformed into rutile $\mathrm{TiO}_{2}$ when the temperature was above $750{ }^{\circ} \mathrm{C}$. The condensation and growth of particles can be observed during the heating process. The key of micro-emulsion methods is to prepare uniform, controllable, and stable micro-emulsions. Micro-emulsion methods own several advantages such as no heating, simple equipment, easy operation, and controlling products' particle sizes, but they require consuming large amounts of organic solvents/surfactants and high-temperature calcination to remove residual organic matters. How to reduce cost, restrain crystalline phase transformation and re-agglomeration is still required to be solved. ${ }^{[23]}$

\section{Sol-gel method}

Hydrolysis of titanium alkoxide is a kind of sol-gel method using titanium alkoxide as a raw material. The raw material goes through hydrolysis and polycondensation to obtain sol, then further polycondensation to get gel. The gel is dried and calcined to obtain nano- $\mathrm{TiO}_{2}$. Music et al. ${ }^{[24]}$ explored the sol-gel process of a ternary system of tetraisopropanolorthotitanate (TTIP)/isopropanol ( $i-\mathrm{PrOH}) /$ concentrated nitric acid. The results presented that adding polyethylene glycol (PEG) into the system could stabilize the sol and avoided the sintering of the particles during the calcination process. So et $a l .{ }^{[25]}$ analyzed the effect of the molar ratio of nitric acid/TTIP on the crystalline phase of the gel. The results indicated that the product was amorphous when nitric acid was not added. When mole ratio of nitric acid/TTIP was low, the product was anatase $\mathrm{TiO}_{2}$. With the increase of the molar ratio, rutile $\mathrm{TiO}_{2}$ appeared and its content increased gradually. Vorkapic et al. ${ }^{[26]}$ found that different types of alkoxide only affected the size of primary particles. The sol particles were the aggregates of the primary particles, the size of which was mainly controlled by the process of peptization. The most important factor was peptization temperature. Huang et al. ${ }^{[27]}$ prepared $\mathrm{TiO}_{2} /$ ethylene glycol slurry at room temperature by controlling the water and metal alkoxide molar ratio from 2 to 4 and the hydrolysis rate of titanium glycol. TEM analysis showed that nano- $\mathrm{TiO}_{2}$ particles with a particle size of 5-10 nm were homogeneously dispersed in ethylene glycol. Chen et al. ${ }^{[28]}$ used butyl titanate as raw materials for preparation of nano- $\mathrm{TiO}_{2}$, and they used XRD to analyze grain size of colloidal $\mathrm{TiO}_{2}$ which was calcined at different temperatures. The results showed that $\mathrm{TiO}_{2}$ particles own anatase structures at calcined temperature of 473 $\mathrm{K}$ and its particle size was about $5.5 \mathrm{~nm}$. When calcined temperature was higher than $673 \mathrm{~K}$, the particle size of $\mathrm{TiO}_{2}$ increased rapidly, and mix-crystal structure of anatase and rutile was formed. $\mathrm{TiO}_{2}$ particles were completely transformed into rutile $\mathrm{TiO}_{2}$ at $973 \mathrm{~K}$. Gopal et $a l .{ }^{[29]}$ hydrolyzed titanium alkoxide to form sol under acidic conditions and then heated at different heating rates. At atmospheric pressure and a heating temperature below $100{ }^{\circ} \mathrm{C}$, anatase nano- $\mathrm{TiO}_{2}$ with particle size of $50-100 \mathrm{~nm}$ was synthesized.

The titanium alkoxide hydrolysis method owns several advantages. Firstly, nano- $\mathrm{TiO}_{2}$ with specific pore structures can be formed by adding an appropriate amount of surfactants or template agents that will make nanoparticles grow in a particular direction. Secondly, the purity of raw materials is high and impurity ions are not introduced in the whole process. Thirdly, reaction conditions are relatively mild. Finally, high purity, small particle size, and narrow size distribution nano- $\mathrm{TiO}_{2}$ can be obtained by controlling process conditions. However, the method suffers from high cost for raw material. Furthermore, the gel will go through volume shrinkage in the drying and calcination process, causing agglomeration of nano- $\mathrm{TiO}_{2}$ particles. $^{[23]}$

Considering grain size distribution, cost, crystal form and other factors, the liquid phase method is mainly employed to prepare nano- $\mathrm{TiO}_{2}$ for cosmetics.

\section{Application of nano- $\mathrm{TiO}_{2}$ in cosmetics}

From the point of view of developmental trend of sunscreen cosmetic, there are two types of sunscreens with great potential including inorganic and bionic sunscreens. It is difficult to promote bionic sunscreen for its high cost presently. However, inorganic sunscreen is more affordable possessing superior sun-protect performances and great market potential. Nano- $\mathrm{TiO}_{2}$ has the best potential among inorganic sunscreens.

\section{Select nano- $\mathrm{TiO}_{2}$ for cosmetics}

\section{Select crystal structures}

Commonly used $\mathrm{TiO}_{2}$ is a square crystal including three crystal forms: anatase, rutile, and brookite. Rutile $\mathrm{TiO}_{2}$ could be obtained by 
calcining anatase $\mathrm{TiO}_{2}$, and its lattice structure is more perfect and thermodynamically more stable. The refractive index of rutile $\mathrm{TiO}_{2}$ is 2.7, while anatase $\mathrm{TiO}_{2}$ is 2.3 . Rutile $\mathrm{TiO}_{2}$ has better UV reflection ability, scattering ability, and weak absorption capacity. In the presence of oxygen, the photo activity of rutile $\mathrm{TiO}_{2}$ is weaker than that of anatase $\mathrm{TiO}_{2}{ }^{[30,31]}$ Generally, the effect of rutile $\mathrm{TiO}_{2}$ shielding $\mathrm{UV}$ is more significant than anatase $\mathrm{TiO}_{2}$, so the mainly used nano- $\mathrm{TiO}_{2}$ in cosmetics is rutile $\mathrm{TiO}_{2}$.

\section{Select particle sizes}

The UV resist ability of nano- $\mathrm{TiO}_{2}$ is closely related to its particle size. When the particle size is equal to or less than half wavelength of incident light, the amount of light reflection and scattering reach its maximum. Hence, the shielding effect is the best. Since the UV ray's wavelength is between 190 and $400 \mathrm{~nm}$, the particle size of nano- $\mathrm{TiO}_{2}$ should be no more than $200 \mathrm{~nm}$, preferably, no more than $100 \mathrm{~nm}$. In addition, particles that are too small are easy to agglomerate, which is not conducive to its dispersion. Tiny particles are also easy to plug the pores on human skin and then affect skin breathable and sweat discharge. The particle size is controlled between 30 and $100 \mathrm{~nm}$. When the particle size is between this range, the UV shielding effect is the best and the visible light can also be transmitted, which bring out more natural beauty of the skin. ${ }^{[32-35]}$

\section{Select preparation methods}

Wet and dry methods are performed to prepare nano- $\mathrm{TiO}_{2}$. The rutile $\mathrm{TiO}_{2}$ synthesized by wet methods owns better dispersibility, but it suffers from impure crystal form and high photocatalytic activity. However, rutile $\mathrm{TiO}_{2}$ obtained by dry methods possesses higher purity and less photocatalytic activity, but its dispersion is poor. ${ }^{[36]}$ Nowadays, the liquid-based methods are widely employed for preparation of nano- $\mathrm{TiO}_{2}$ in cosmetics considering crystal form, particle size distribution, cost, and other factors. $\mathrm{TiOSO}_{4}$ or $\mathrm{TiCl}_{4}$ is used as a raw material to prepare a solution with a certain concentration, then alkaline solution is added to obtain $\mathrm{TiO}_{2}$ hydrate by hydrolysis. After depolymerization, washing, drying and calcinations, nano- $\mathrm{TiO}_{2}$ can be produced. $\mathrm{TiO}_{2}$ with different particle size and crystal form can be obtained by changing calcination temperatures. ${ }^{[32]}$

\section{Sun protection mechanism of nano- $\mathrm{TiO}_{2}$}

Nano- $\mathrm{TiO}_{2}$ protects the skin by reflecting and scattering UV irradiation. ${ }^{[37,38]}$ The surface effect of nano- $\mathrm{TiO}_{2}$ can cause surface atoms of nanoparticles to deform, changes in electron spin conformation and electron spectroscopy, and new photochemical properties. The quantum size effect of nano powders results in "blue-shift phenomenon" at the absorption of a certain wavelength light and "broadening phenomenon" at the absorption of various wavelengths light, and a significant enhancement of the absorption effect of UV light. ${ }^{[39]}$ The size of nano- $\mathrm{TiO}_{2}$ particle is less than $100 \mathrm{~nm}$, so it can effectively absorb and scatter UV light. When UV light irradiates nano- $\mathrm{TiO}_{2}$ particles, the electrons of the nanoparticles are forced to vibrate (the frequency of the vibration is the same as that of the incident light). The electrons become secondary wave source, and emit electromagnetic waves in all directions, and that is the scattering of ultraviolet light. In addition, $\mathrm{TiO}_{2}$ is an $n$-type semiconductor. Anatase $\mathrm{TiO}_{2}$ 's band gap width is $3.2 \mathrm{eV}$, while rutile $\mathrm{TiO}_{2}$ 's band gap width is $3.0 \mathrm{eV}$. The electrons on the valence band can absorb UV light, be excited to the conduction band, and generate electron-hole pairs at the same time. That is why $\mathrm{TiO}_{2}$ can absorb UV light. ${ }^{[6,32,40-43]}$

\section{Problems of nano- $\mathrm{TiO}_{2}$ as sunscreen in cosmetic}

\section{Aggregation problems}

Smaller the size of nano- $\mathrm{TiO}_{2}$ particles, stronger the interactions between particles, such as electrostatic attraction force, van der Waals force and capillary force, which will cause aggregation of particles. As nanoparticles have a large specific surface area, the surface energy is also very large so the system will be in a relatively unstable thermodynamic state. Particles will aggregate to reduce free enthalpy of the system, which is an unavoidable spontaneous process. The instability of nano- $\mathrm{TiO}_{2}$ limits its application in cosmetics greatly.

\section{Photocatalytic activities}

Nano- $\mathrm{TiO}_{2}$ has excellent photocatalytic activities. Under light irradiation, it can catalyze some chemical reactions. Nano- $\mathrm{TiO}_{2}$ directly used in cosmetics will decompose fragrances/nutrients and oxidize grease in cosmetics, causing deterioration of cosmetics. In addition, nano- $\mathrm{TiO}_{2}$ may damage human skin. In clinical trials, $\mathrm{TiO}_{2}$ can cause severe human skin tissue necrosis and non-specific dermatitis, which can lead to deposition of melanin in the skin. ${ }^{[6,44]}$

\section{Hydrophilic and oleophobic properties}

As a kind of inorganic additive, nano- $\mathrm{TiO}_{2}$ is difficult to disperse in an organic medium, which limits its application in cosmetics. It is necessary to modify nano- $\mathrm{TiO}_{2}$ and increase its compatibility with organic medium. ${ }^{[45-47]}$

\section{Surface modification of nano- $\mathrm{TiO}_{2}$}

In view of the problems existing in the application of nano- $\mathrm{TiO}_{2}$ in sunscreen products, researchers hope to improve its safety and dispersity by applying the surface modifications. The surface modified methods of nano- $\mathrm{TiO}_{2}$ include inorganic and organic treatments. ${ }^{[48-54]}$

\section{Inorganic treatments}

Inorganic treatment of nano- $\mathrm{TiO}_{2}$ is depositing inorganic compounds or metals on the $\mathrm{TiO}_{2}$ particles (by certain methods) to form a covering film or build a core-shell structure particle. Then the covered particles will be treated by washing, dehydrating, drying, and calcining, so that the covering film could firmly attach to the particle surface. Many inorganic treating agents are used to improve surface properties of nano- $\mathrm{TiO}_{2}$ particles such as oxides of aluminum, zirconium, silicon, zinc, chrome, tin, etc. By coating the nanoparticles with inorganic materials, less UV irradiation is able to reach $\mathrm{TiO}_{2}$, which leads to less UV light absorption for $\mathrm{TiO}_{2}$ particles. Meanwhile, this treatment can also change the crystal structure of the particles' surface layerand, consequently, change the particles' electrochemical behavior. The covering materials can be used as an effective barrier to restrain the photocatalytic activity of the $\mathrm{TiO}_{2}$, increase the dispersion ability of the nano- $\mathrm{TiO}_{2}$ particles, and reduce direct contact between skin and harmful groups on the surface of the particle. ${ }^{[29]}$ In inorganic treatment, if only one kind of coating agent is used, the modification effect is limited. For example, performance of nano- $\mathrm{TiO}_{2}$ coated with aluminum or silicon alone is not as good as co-coated with aluminum and silicon. ${ }^{[55]}$ Wei et al. ${ }^{[56]}$ used sol-gel method to incorporate silver into $\mathrm{TiO}_{2}$, and used silver-loaded $\mathrm{TiO}_{2}$ as raw material to prepare antimicrobial sunscreen cream, then they have studied the effect of silver-loaded $\mathrm{TiO}_{2}$ on sun protect performance, color stability and antibacterial property of the cream. The results showed that silver-loaded in $\mathrm{TiO}_{2}$ can promote the transformation of crystal structure from anatase to rutile and improve the sun protection effect. When silver-loaded $\mathrm{TiO}_{2}$ was used as a skin cream or sunscreen, the high silver content significantly affects the color of the product. In addition, silver-loaded $\mathrm{TiO}_{2}$ can fully meet the cosmetics' antibacterial requirement. ${ }^{[57,58]}$

\section{Organic treatments}

Nano- $\mathrm{TiO}_{2}$ with hydrophilic surface can be obtained by inorganic treatments and it is suitable for using in polar solvents. However, hydrophilic particles are difficult to disperse in organic systems such as oily cosmetics and they can't demonstrate special functions of nano- $\mathrm{TiO}_{2}$. In order to improve compatibility and dispersibility of nano- $\mathrm{TiO}_{2}$ in organic systems, organic surface treatment can be carried out. Organic treatment agents included surfactants, coupling 
agents, and organic polymers. ${ }^{[59,60]}$

\section{Surfactants}

Surfactants include anionic, cationic, and nonionic surfactants. One end of surfactant molecules is a long-chain alkyl group, and another end is a carboxyl group, ether group, amino group, or other polar groups. Surfactants can be physically or chemically absorbed on the surface of nano- $\mathrm{TiO}_{2}$ particles using the polar groups. ${ }^{[33,55]}$

Zou et al. ${ }^{[61]}$ prepared stearic acid surface modified nanoparticles in a mixed solvent by a sol-gel method., which were characterized by FT-IR, XPS, XRD, and TEM. They confirmed organic coating on the surface of the nanoparticles and carboxylate groups in the stearic acid molecule bounded to inorganic core in bidentate coordination. Li et $a l .^{[62]}$ synthesized oleic acid-modified nanoparticles by surface modification method. The surface modified nanoparticles were characterized by IR, TEM and XPS, and the effect of oleic acid concentration on the surface coverage and the dispersibility of modified nanoparticles in oil was further investigated. The results demonstrated that after the surface modification by oleic acid, the nano- $\mathrm{TiO}_{2}$ with well dispersion performance in the oil phase was successfully prepared. Jiang et $a l .{ }^{[63]}$ used stearic acid and adipic acid as a modification agent; they modified the surface of nanoparticles by a sol-gel supercritical drying method, and well solved the dispersion problem of nanoparticles in organic medium. It was found that the growth and aggregation of nanoparticles were controlled because the presence of the surface modification layer. Zhao et al. ${ }^{[64]}$ successfully synthesized the surfactant coated $\mathrm{TiO}_{2}$ particles by hydrolyzing titanate in the water center of W/O type micro emulsion, which was comprised of water/Span-80 (or DBS) or $n$-amyl alcohol/cyclohexane. Li et al. ${ }^{[65,66]}$ modified nanoparticles using organic silanes, titanate coupling agents, sodium dodecyl benzene sulfonate, and sodium laurate. In terms of the lipophilic degree of nanoparticles, they found that the best surface treatment agent was sodium laurate. They discussed the mechanism of nano surface modification and the optimization of modification process conditions. The change of the bonding state and particle size of nanoparticles before and after modification was also studied. Nussbaumer et al. ${ }^{[67]}$ selected dodecyl benzene sulfonic acid as an organic modifying agent for surface modification of rutile nano- $\mathrm{TiO}_{2}$. The resulting product was almost transparent in toluene, but it could absorb ultraviolet light over a wide range of wavelengths. ${ }^{[23]}$

\section{Coupling agents}

Coupling agents are substances with amphipathic structures, and their functional groups can react with active groups on the surface of nano powders to form strong chemical bonds. Other functional groups can interact with molecules of organic polymers to cause chemical reactions or physical entanglements. As a result, nano- $\mathrm{TiO}_{2}$ particles and organic media produce a special function molecular bridges that improve comprehensive performances of nanocomposites. ${ }^{[23,68,69]}$ The coupling agents are involved in silicates, titanates, aluminates, etc.

Li et al. ${ }^{[70]}$ pretreated nano- $\mathrm{TiO}_{2}$ particles with butyl titanate firstly, and then modified them using methyl methacrylate (MMA) as a coupling agent. The results indicated that the reaction between butyl titanate and $\mathrm{TiO}_{2}$ surface hydroxyl groups resulted in the formation of crosslinking reactants on the surface of the particles. The further reaction of crosslinking reactants with methyl methacrylate caused the formation of polymethylmethacrylate (PMMA) and the PMMA uniformly coating on the surface of $\mathrm{TiO}_{2}$ nanoparticles. The nanoparticles modified by polymerization own good dispersibility in toluene. Deusser et al ${ }^{[71]}$ utilized two kinds of organosiloxanes for dry surface treatment of nanosized $\mathrm{TiO}_{2}(\mathrm{P} 25)$, and Kerner et al. ${ }^{[72]}$ treated nano $\mathrm{TiO}_{2}$ with amino-containing silanes. Their results showed that the treated nanopowders could be well dispersed in hydrophilic and lipophilic medium. ${ }^{[73,74]}$

In addition, other literatures presented that nano- $\mathrm{TiO}_{2}$ composite particles with uniform dispersion, high stability and good UV protection could be obtained by coupling agent treatment. ${ }^{[75,76]}$

\section{Polymer coating method}

The van der Waals force between particles can be reduced by coating polymers on the surface of $\mathrm{TiO}_{2}$. Moreover, a new repulsion force, which is steric resistance, is produced. It is helpful to enhance the compatibility between nano- $\mathrm{TiO}_{2}$ composites and organic matrices and improve the nano- $\mathrm{TiO}_{2}$ dispersibility in organic medium. Polymer coating methods include a pretreatment method and a direct coating method. ${ }^{[77]}$

Due to the presence of free radicals and positive/negative ions on the surface of $\mathrm{TiO}_{2}$, the direct coating method can directly lead to monomer polymerization. As to the pretreatment method, it is difficult for organic monomers and polymers to be adsorbed on the surface of particles due to the strong polarity on the surface of $\mathrm{TiO}_{2}$, resulting in low treatment efficiencies. Pre-treatment with silane coupling agents, surfactants, polymers, and so on, is usually performed firstly to reduce surface activity, then followed by polymer coating modification on the surface of $\mathrm{TiO}_{2}{ }^{[77,78]}$

Zhang et al. ${ }^{[79]}$ prepared $\mathrm{TiO}_{2}$ coated with polymethylmethacrylate (PMMA) using in-situ emulsion polymerization. They found that nano- $\mathrm{TiO}_{2}$ did not inhibit the polymerization of methyl methacrylate. The results suggested that the coating of PMMA on the surface of nano- $\mathrm{TiO}_{2}$ was mainly covered physically, while chemical coating was little. Tan et al. ${ }^{[80]}$ used sodium dodecyl sulfate for pre-modification of $\mathrm{TiO}_{2}$ particles, and then the pre-modified particles were employed as nucleus. Methyl methacrylate and styrene were used as monomers to coat $\mathrm{TiO}_{2}$ by the polymerization method to obtain $\mathrm{TiO}_{2}$-polymer compounds encapsulated particles with good surface coating properties. ${ }^{[77]} \mathrm{Xu}$ et al. ${ }^{[81]}$ used toluene 2,4-diisocyanate (TDI) as wall materials by the interface polymerization method under different conditions to prepare a series of $\mathrm{TiO}_{2}$ polyurea microcapsules. It was found that the density and hydrophilicity of polyurea microcapsules decreased greatly, and its affinity with ethonal was greatly improved.

\section{Conclusions}

Nano- $\mathrm{TiO}_{2}$ has received the favor of sunscreen cosmetics producers for its unique properties of scattering and reflection of UV. Though preparation and modification of nano- $\mathrm{TiO}_{2}$ is successful, its theoretical and application is not satisfactory. Therefore, the industrial application of nano- $\mathrm{TiO}_{2}$ in sunscreen cosmetics is still limited. ${ }^{[56]}$ How to ensure the strong absorption UV property of nano- $\mathrm{TiO}_{2}$, weaken or eliminate its photocatalytic activity, and improve its dispersibility in cosmetics, is an urgent problem that needs to be solved in the future.

\section{Acknowledgement}

The work was supported by the National Fund Cultivation Project (NFC 15001), the Subject and Specialty Construction Special Fund of Guangdong Provincial Higher Education Institution (Nos. 2012CXZD0024 and 2013KJCX0081), and the Science and Technology Plan Project of Guangdong Province (No. 2013B030600001).

\section{References}

[1] Liu, Y. M.S. Dissertation, Wuyi University, Guangdong, 2006 (in Chinese).

[2] Li, S. Y.; Lu, K.; Ruan, H. J. J. Hyg. Res. 2001, 30, 319.

[3] Li, X. E.; Fan, A.; Wang, X.; Zu, Y. Paint Coat. Ind. 2000, 3.

[4] Yu, A. P.; Chen, Z. W.; Chen, X. H.; Fang, T. N.; Gu, H. Ch. Mater. Rev. 2001, 15, 38 .

[5] Zhao, Z. Q. J. Environ. Health 1999, 16, 245.

[6] Chen, Q. Y. Preparation, Characterization and Safety Application of Nanometer Titanium Dioxide, Beijing, Science Press, 2014, 3.

[7] Li, G. H.; Li, C. Z.; Zhu, Y. H. Chem. World 2000, 59 
[8] Peng, J. Y.; Li, Z. Y.; Chen, L. C.; Wen, W. Z.; Hu, J. Q. Guangdong Chem. Ind. 2015, 42, 115.

[9] Zu, Y.; Lei, Y. Y.; Yu, H. New Chem. Mater. 1998, 26.

[10] Wang, R. M.; Du, J. X.; Liu, F.; Chen, L.; Liu, R. X.; Ge, G. L. Chin. J. Anal. Lab. 2016, 35, 1278.

[11] Xie, S. L.; Gou, J. X.; Jia, D. M.; Zhang, J.; Zheng, X. D. Chem. Ind. Times 2008, 22, 66 .

[12] Meng, Q.; Liu, X. H.; Wang, Z.; Wang, H. Y. Ind. Sci. Tribune 2016, 15, 78.

[13] Yin, T. M.S. Dissertation, Chongqing University, Chongqing, 2011 (in Chinese).

[14] Gui, L. L. Technol. Wind 2011, 53.

[15] Seifried, S.; Winterer, M.; Hahn, H. Chem. Vapor Depos. 2000, 6, 239.

[16] Meng; Q.; Liu, X. H.; Wang, Zh.; Wang, H. Y. Ind. Sci. Tribune 2016, 15, 78.

[17] Hong, S. S.; Man, S. L.; Lee, G. D.; Lim, K. T.; Ha, B. J. Mater Lett. 2003, 57, 2975.

[18] Nad, S.; Sharma, P.; Roy, I.; Maitra, A. J. Colloid Interface Sci. 2003, $264,89$.

[19] Chen, X.; Mao, S. S. Chem. Rev. 2007, 107, 2891.

[20] Lin, J.; Lin, Y.; Liu, P.; Mohammed, J. M.; Lawrence, F. A.; Sun, Y. P. J. Am. Chem. Soc. 2002, 124, 11514.

[21] Kim, C. S.; Moon, B. K.; Park, J. H.; Choi, B. C.; Seo, H. J. J. Cryst. Growth 2003, 257, 309.

[22] Li, G. L.; Wang, G. H. Nanostruct. Mater. 1999, 11, 663.

[23] Yao, Ch. Ph.D. Dissertation, Nanjing University of Science and Technology, Jiangsu, 2005 (in Chinese).

[24] Musić, S.; Gotić, M.; Ivanda, M.; Popović, S.; Turković, A.; Trojko, R.; Sekulić, A.; Furić, K. Mater. Sci. Eng. B 1997, 47, 33.

[25] So, W. W.; Park, S. B.; Moon, S. J. J. Mater. Sci. Lett. 1998, 17, 1219.

[26] Vorkapic, D.; Matsoukas, T. J. Am. Ceram. Soc. 2005, 81, 2815.

[27] Huang, W.; Wu, Y.; Gu, Y. H.; Gu, H. Ch. J. East China Univ. Sci. Technol. 2003, 29, 198.

[28] Chen, Q. L.; Tang, C. Q.; Xiao, X. Ding, S. F. J. Mater. Sci. Eng. 2002, $20,224$.

[29] Gopal, M.; Chan, W. J. M.; De Jonghe, L. C. J. Mater. Sci. 1997, 32, 6001.

[30] Du, H. Beijing Daily Chem. 2001, 16.

[31] Zu, Y.; Lei, Y. Y.; Fan, A. Titanium Ind. Prog. 1998, $21,28$.

[32] Xu, C. Y.; Duan, Y. B. Yunnan Chem. Technol. 2004, 31, 36.

[33] Duan, L. M.; Ma, J. Zh.; Lv, B.; Sun, H. Y. China Leather 2015, 44, 39.

[34] Lu, N. H.; Zhu, Zh. N.; Zhao, X. Q.; Tao, R.; Yang, X. L.; Gao, Zh. H. Biochem. Biophys. Res. Commun. 2008, 370, 675.

[35] Yang, Y.; Han, A. J.; Luo, F. Sh.; Li, F. S. Flavour Frag. Cosmetics 2001, 14.

[36] Gonzalez, G. H.; Farbrot, A.; Larkö, O. Clin. Exp. Dermatol. 2002, 27, 691.

[37] Yu, Sh. J.; Zheng, Y. B.; Du, J.; Liu, J. P.; Shang, H. Zh.; Liu, L. China Surfactant Deterg. Cosmetics 2005, 35, 248.

[38] Zhao, X. M.; Chen, Zh. H.; Li, Y. N. Chin. J. Aesthet. Med. 2015, 24, 78.

[39] Chen, C. H. M.S. Dissertation, Taiyuan University of Technology, Shanxi, 2008 (in Chinese).

[40] Yao, C.; Zhang, Z. H.; Lin, X. P.; Wang, X. China Surfactant Deterg. Cosmetics 2003, 33, 333.

[41] Zhang, J. Y. Ph.D. Dissertation, Shanghai Jiaotong University, Shanghai, 2012 (in Chinese).

[42] Zhang, K. D. M.S. Dissertation, South China University of Technology, Guangdong, 2014 (in Chinese).

[43] Cao, Zh.; Zhang, D. J. China Surfactant Deterg. Cosmetics 2014, 44, 700 .

[44] Boisnic, S.; Branchet, G. M. K. C.; Nocera, T. Skin Pharmacol. Physiol.
2005, 18, 201.

[45] Tsubokawa, N.; Kimoto T.; Endo T. Polym. Bull. 1994, 33, 187.

[46] Zhao, J. L.; Zhou, F. C.; Jin, Ch. M.; Zhang, X. Q.; Piao, Zh. S.; Gao, Y.; Huang, Sh. H.; Yu, J. Q.; Zou, B. S.; Zhang, Y.; Xiao, L. Zh. Chin. J. Chem. Phys. 1993, 6, 129.

[47] Wu, L. D. M.S. Dissertation, South China University of Technology, Guangdong, 2011 (in Chinese).

[48] LU, H. R. M.S. Dissertation, Suzhou University, Jiangsu, 2010 (in Chinese).

[49] Wang, L. F. M.S. Dissertation, Huazhong University of Science and Technology, Hubei 2006 (in Chinese).

[50] Zhang, J. X. M.S. Dissertation, Huazhong University of Science and Technology, Hubei, 2005 (in Chinese).

[51] Hao, X. M.; Li, H. M.; Li, F.; Shi, C. P.; Sun, M. Inorg. Chem. Ind. 2012, 44,30 .

[52] Guo, L. Y. M.S. Dissertation, Donghua University, Shanghai, 2015 (in Chinese).

[53] Cui, A. L.; Wang, T. J.; Jin, Y. Chem. J. Chin. Univ. 2000, 21, 1560.

[54] Zhang, Z.; Zhong, J. J.; Liu, J. H. Leather Chem. 2013, 30, 15.

[55] Xu, L.; Geng, J. Y.; Su, Y.; Huang, W. Heilongjiang Sci. 2016, 7, 26.

[56] Wei, S. W.; Li, Z. Y. Fine Spec. Chem. 2011, 19, 17.

[57] Yu, Y. H.; Hari, B. L.; Xu, C. Y. Inorg. Chem. Ind. 2008, 40, 11.

[58] Liu, C. L. Liaoning Chem. Ind. 2008, 37, 152.

[59] Li, G. H.; Li, Ch. Zh.; Zhu, Y. H. Chem. World 2000, 59.

[60] Luo, G. D.; Yu, Q.; Tan, X. K.; Ke, Y. L.; Yu, L.; Chen, L.; Zhang, Z. H.; Luo, Y. H. Chem. World 2016, 533.

[61] Zou, L.; Wu, X. D.; Chen, H. G.; Wang, D. P. Acta Phys.-Chim. Sin. 2001, $17,305$.

[62] LI, Z. W.; Zhu, Y. F. Acta Chim. Sinica 2003, 61, 1484.

[63] Jiang, X. M.; Wang, X. Y.; Chen, Y. Z. Acta Petrolei Sinica 2002, 8, 61.

[64] Zhao, X. P.; Yin, J. B.; Xiang, L. Q. Chin. J. Mater. Res. 2001, 15, 308.

[65] Li, X. E.; Deng, H.; Fan, A.; Ren, Y. X.; Zu, Y. J. Northwest Univ. 2002, $32,523$.

[66] Li, X. E.; Deng, H.; Zhang, F. Y.; Fan, A.; Ren, Y. X.; Wen, H. M.; Zu, Y. Inorg. Chem. Ind. 2001, 33, 5.

[67] Nussbaumer, R. J.; Caseri, W.; Tervoort, T.; Smith, P. J. Nanopart. Res. 2002, 4, 319.

[68] Ding, H.; Lu, S. C.; Zhang, K. R.; Zhang, Y. N. Conserv. Util. Miner. Resour. 1996, 25.

[69] Qi, X. k.; Wang, X. X.; Qu, J. M.; Yang, H.; Fan, W. H.; Zou, T. Mater. Rev. 2015, 29, 5.

[70] Li, G. H.; Li, Ch. Zh.; Lv, Z. M. J. East China Univ. Sci. Technol. 2000, 26,639 .

[71] Deusser, H.; Kerner, D.; Meyer, J.; Michael, G.; Stubbe, A. US 5372905 , 1994.

[72] Deller, K.; Kerner, D.; Meyer, J. DE 19929845, 2001

[73] Zheng, Y. J. M.S. Dissertation, Hebei University, Hebei, 2007 (in Chinese).

[74] Yao, Ch.; Yang, G.; Lin, X. P.; Wang, X. China Surfactant Deterg. Cosmet. 2004, 34, 252.

[75] Chen, Y. F.; Li, L. M.; Yuan, G. X.; Yue, W.; Xiao, Y. Y. Nat. Sci. J. Harbin Normal Univ. 2011, 27, 71.

[76] Chen, Q. Y. Inner Mongolia Petrochem. Ind. 2008, 4.

[77] Liu, Y. M. Ph.D. Dissertation, Jiangsu University, Jiangsu, 2011 (in Chinese).

[78] Wu, W.; Chen, J. F.; Lu, S. C. Superfine Powder Surface Modification, Chemical Industry Press, Beijing, 2004.

[79] Zhang, L. J.; Chen, J. L.; Zhao, Z. Y. Paint Coat. Ind. 2003, 33, 1.

[80] Tan, D. S.; Yan, N. X.; Shi, Y. J. Polym. Mater. Sci. Eng. 1999, 15, 101.

[81] Xu, D. M.; Zhang, K. D.; Wang, P.; Fan, Z. H. Fine Chem. 2002, 19, 25. 